\title{
Periostin Is a Novel Factor in Cardiac Remodeling After Experimental and Clinical Unloading of the Failing Heart
}

\author{
William E. Stansfield, MD, Nancy M. Andersen, MD, Ru-Hang Tang, PhD, and Craig H. \\ Selzman, MD \\ Division of Cardiothoracic Surgery, Department of Surgery, University of North Carolina, Chapel \\ Hill, North Carolina; and Division of Cardiothoracic Surgery, University of Utah, Salt Lake City, \\ Utah
}

\begin{abstract}
Background-Maladaptive left ventricular hypertrophy (LVH) remains a prevalent and highly morbid condition associated with end-stage heart disease. Originally evaluated in the context of bone development, periostin is important in endocardial cushion formation and has recently been implicated in heart failure. Because of its potential role in cardiovascular development, we sought to establish the role of periostin after relief of pressure overload in animal and human models.
\end{abstract}

Methods-Pressure overload induction of LVH was performed by minimally invasive aortic arch banding of C57Bl6 mice. Bands were removed 1 month later to allow regression. Cardiac tissue was procured in paired samples of patients receiving LV assist devices (LVAD), with subsequent reanalysis at the time of explant for transplantation.

Results-One week after debanding, heart weight/body weight ratios and echocardiography confirmed decreased LV mass relative to hypertrophied animals. Gene and protein expression of periostin was measured by real-time polymerase chain reaction and Western blot, and was similarly decreased compared with LVH mice. Immunohistochemical localization of periostin showed it was exclusively in the extracellular matrix of the myocardium. The decrease in periostin with pressure relief paralleled changes in interstitial fibrosis observed by picrosirius red staining. Corroborating the murine data, periostin expression was significantly reduced after LVADafforded pressure relief in patients.

Conclusions-Periostin is closely associated with pressure overload-induced LVH and LVH regression in both animal and human models. The magnitude of expression changes and the consistent nature of these changes indicate that periostin may be a mediator of cardiac remodeling.

Left ventricular hypertrophy $(\mathrm{LVH})$ remains a highly prevalent and a powerful independent risk factor for cardiovascular death and disease in North America [1]. Initially viewed as beneficial, LVH transitions to a maladaptive phase and leads to clinical heart failure.

Although many have studied the remodeling process, few have focused on the changes that occur when a failing heart recovers.

We recently described the physiologic and genomic changes that occur in a murine model of pressure overload and subsequent relief [2]. First, we demonstrated that increased LV mass associated with aortic constriction is largely reversible. Second, during the processes of

(C) 2009 by The Society of Thoracic Surgeons Published by Elsevier Inc

Address correspondence to: Dr Selzman, Division of Cardiothoracic Surgery, University of Utah, 30 N 1900 E, SOM 3C 127. Salt Lake City, UT 84132; craig.selzman@hsc.utah.edu.

Presented at the Poster Session of the Forty-fifth Annual Meeting of The Society of Thoracic Surgeons, San Francisco, CA, Jan 2628, 2009. 
hypertrophic progression and regression, we identified several dominant functional groups of genes that were independently expressed during LVH progression and regression. Notably, we observed an abundance of genes that control the development and function of the extracellular matrix (ECM). One gene in particular, periostin, stood out from this group for its previous genomic associations with both ventricular hypertrophy [3] and heart failure [4].

Periostin is an ECM protein that derived its name from studies of bone development in which it localized to both cortical bone periosteum and the periodontal ligament. Its first association with the heart was described in the context of endocardial cushion formation and heart valve formation [5]. Subsequently, periostin has been identified as a secreted factor within the ECM. It is believed to have a strong relationship with fibrosis and ECM metabolism in a variety of disease states, including cardiovascular disease and cancer [6].

In this context, we sought to determine the role of periostin in cardiac remodeling. Using our existing models for pressure overload and relief in both mice and humans, we demonstrate that periostin expression and patterns of fibrosis are closely related to pressure overload, and that in suitable conditions, periostin expression, $\mathrm{LVH}$, and fibrosis can be significantly reversed.

\section{Material and Methods}

\section{Surgical Model and Experimental Design}

In accordance with both an institutionally approved Institutional Animal Care and Use Committee protocol and National Institutes of Health guidelines, 10-week-old C57B16 male mice $(n=60)$ were randomly assigned to one of three groups: sham, band, and deband (Fig. $1)$.

Mice in the sham and band groups underwent minimally invasive transverse aortic banding as previously described [7]. Briefly, animals were anesthetized using inhaled isoflurane by face mask. A midline neck incision was used to approach the anterior mediastinum. The transverse arch was identified, and a constrictive band was placed and tightened to the approximate diameter of a 27-gauge needle. The only difference between sham and band groups was that the constrictive band was not tightened in the sham animals. Adequate placement of the band was verified by evaluation of carotid Doppler imaging before and after placement of the aortic band. Adequate banding was accepted when the Doppler velocity ratio doubled from right to left carotid arteries.

Animals in the deband group underwent an initial procedure identical to the band animals. At 4 weeks, the aortic band was removed [7]. Efficiency of debanding was verified by carotid Doppler with normalization of carotid velocities. Deband and sham deband animals were sacrificed 1 week after the deband procedure for all evaluations except histology, where animals were sacrificed both at 1 and 4 weeks after debanding.

\section{Transthoracic Echocardiography}

Transthoracic echocardiography was performed using the Vevo 660 High Resolution Biomicroscopy System equipped with a 30-Mhz transducer (Visual Sonics, Toronto, ON, Canada). During examination, mice were anesthetized with $1 \%$ to $1.5 \%$ inhaled isoflurane. Depth of anesthesia was standardized by recording images at heart rates of 480 to 520 beats/ min. Images were recorded in all animals preoperatively, at 2 and 4 weeks after banding, and at 1 week after debanding. Two technicians, blinded to the animals' experimental status, performed examinations and measurements. 


\section{Mouse Tissue Procurement}

At time of sacrifice, hearts were rapidly excised and the LV apices were sectioned and flash frozen in liquid nitrogen for gene array analysis. Additional animals allocated to histologic analysis were perfused with phosphate-buffered saline, followed by $10 \%$ formalin, fixed overnight in formalin, and then processed for histologic analysis using periodic acid Schiff staining. The cardiomyocyte cross-sectional area was measured using ImageJ $1.38 \mathrm{j}$ software (National Institutes of Health, Bethesda, MD) [8].

\section{RNA Preparation and Real-Time Polymerase Chain Reaction}

LV apices were homogenized in $0.5 \mathrm{~mL}$ of ice-cold Trizol solution (Sigma, St Louis, MO) using a bead mill homogenizer (Retsch, Newtown, PA). RNA was isolated using the standard Trizol procedure, with additional steps for removal of DNA and fibrous tissue. Purity of RNA was verified by using a 260/280 ratio of 1.95 or higher. An Agilent BioAnalyzer 2100 instrument (Agilent Technologies Inc, Santa Clara, CA) was used to verify RNA integrity for all samples. Real-time polymerase chain reaction (PCR) was performed using TaqMan primers and probes (Applied Biosystems International, Foster City, CA).

\section{Western Blot}

Protein fractions were isolated in ice-cold lysis buffer during Dounce homogenization. Concentrations were determined using the Bradford assay. Protein fractions were denatured in loading buffer, and $30 \mu \mathrm{g}$ of each sample was loaded into alternating lanes for gel electrophoresis. Membrane transfer was performed overnight, and rabbit antimouse antibody was used to probe for periostin. Glyceraldehyde-3-phosphate dehydrogenase was used as the loading control.

\section{Immunohistochemistry}

Mouse hearts were perfused with formalin and ethanol at the time of euthanasia, fixed, and then histologically processed according to institution routine, including picrosirius red staining. Formalin-fixed slide blanks were stained with monoclonal antibody to periostin and developed with $3,3^{\prime}$-diaminobenzidine for light microscopic evaluation. Slides were scanned using a ScanScope CS slide scanner using a $\times 40$ objective (Aperio, Vista, CA). Images were cropped to remove valvular tissue and were processed using ImageJ 1.4.1 software. Color thresholding was completed using a custom Java script.

\section{Human Tissue Procurement}

Human myocardial tissue specimens were obtained from patients with end-stage heart disease who were on the transplant waiting list and required LV assist device (LVAD) support. Procurement protocols were approved by the University of North Carolina Institutional Review Board, and patients were appropriately consented before procurement. Briefly, LV apical cores were immediately passed off the table, cut into several specimens, and frozen in liquid nitrogen. At the time of subsequent transplantation, LV specimens from the explanted hearts were similarly cut and frozen in liquid nitrogen. Processing of these tissues was performed as described above.

\section{Statistical Methods}

All physiologic data are presented as mean \pm standard error (SE), except where noted. Realtime PCR data were log-transformed before comparison. All comparisons of physiologic data were performed using two-tailed, type 3 or type $1 t$ tests. Significance was established at the $95 \%$ confidence interval (CI). 


\section{Results}

\section{Periostin Expression Increases With Hypertrophy and Decreases With Regression}

We have previously demonstrated the utility of minimally invasive transverse aortic arch banding and debanding to characterize LVH progression and regression $[2,7]$. To functionally validate our periostin studies, we used transthoracic echocardiography to demonstrate within this cohort of animals that banding caused pressure-induced LVH that was reversed 1 week after debanding (Table 1).

Real-time PCR was used to verify expression levels of periostin in cardiac tissue before and after induction of pressure overload, and after relief of pressure overload (Fig. 2). Comparatively, pressure overload was associated with a 20 -fold increase in expression over baseline (19.9 \pm 7.1 vs $1.20 \pm 0.34, p<0.05)$, and after 1 week of relief from overload, ventricular expression was significantly decreased to only a 5-fold increase from baseline $(4.94 \pm 1.25$ vs $19.9, p<0.05)$. This rise and fall in periostin expression with pressure overload and relief correlates with previously documented changes in the hypertrophic markers $\beta$-myosin heavy chain and skeletal muscle $a-1$ actin. Notably, the deband group did express greater amounts of periostin than the sham group.

Western blot analysis of periostin protein expression demonstrated a similar trend: increased expression with pressure overload was subsequently decreased after relief of pressure overload. Densitometry confirmed significantly increased quantities of periostin in the pressure overload animals relative to baseline $(38,300 \pm 5,600$ vs $20,300 \pm 700, p<0.05)$. Levels of periostin were significantly less in the relief group $(18,300 \pm 5,000$ vs $38,300, p<$ $0.05)$. Unlike the trends with gene expression, differences between the sham and deband groups were not evident at the protein level.

Immunohistochemical analysis was used to identify periostin in both hypertrophied and regressed animals. Sample cross-sectional images demonstrated expression trends similar to those seen with real-time PCR. There was a significant increase in periostin deposition with hypertrophy that decreased with regression. As with the real-time PCR, periostin staining was greater in the deband group relative to the sham group. Curiously, periostin staining was most prominent in the interventricular septum.

\section{Periostin and Fibrosis}

Picrosirius red staining revealed the pattern of collagen deposition in hypertrophy and regression mimicked that observed with periostin (Fig. 3). At the level of the whole heart in cross-section, collagen was increased in pressure overload and decreased in relief of pressure overload. As with periostin, there was a predominance of collagen staining in the interventricular septum relative to the other parts of the heart and LV. Automated image analysis of whole cross-sections of heart was used to quantify this finding, with pressure overloaded hearts demonstrating approximately 2.5 times as much collagen as those of sham or relief hearts. No significant difference in collagen staining was observed between regressed and sham hearts when automated analysis techniques were used.

Higher magnification showed that the bulk of the increased staining of collagen and periostin occurred in both perivascular regions and in the myocardial interstitium (Fig. 3C) Slides scanned using a $\times 20$ objective showed both collagen and periostin staining strongly in the media and adventitia, and then radiating outward into the interstitium. Although minimal staining occurred in the perivascular space of sham specimens, similarly sized vessels in pressure overload specimens showed dramatic increases. In relief of pressure overload, collagen and periostin were both less present in those same locations, with no apparent redistribution of staining, although still more prominent than in sham specimens. 


\section{Periostin is Overexpressed in the Failing Human Heart}

To evaluate our findings in the context of a human model, we used pressure-overloaded hearts from patients with heart failure who were undergoing LVAD placement (Jarvik 2000, Jarvik Heart, Inc, New York, NY). Cardiac tissue was obtained at time of LVAD placement and at time of transplant. Patients all had stage IV heart disease and were status IB on the transplant wait list. The mean age was 40 , with one 21 -year-old outlier. Two patients had idiopathic cardiomyopathy, 1 patient had ischemic cardiomyopathy, and another had a familial cardiomyopathy. All patients had echocardiographically determined ejection fractions of 0.10 to 0.20 before and after VAD placement.

One patient died of a hemorrhagic stroke before transplant, but survived for organ donation; post-VAD cardiac tissue was obtained at this time. The mean time with VAD was $131 \pm 115$ days (range, 32 to 296 days).

All pairs of samples indicated significantly decreased periostin expression by Western blot after mechanical offloading (Fig. 4). The only exception was the patient with ischemic cardiomyopathy who had approximately equivalent expression, and who waited the longest for transplant at 296 days. The 3 patients who received a transplant are all alive at the time of this report, with a mean survival greater than 2 years.

\section{Comment}

Clinically, myocardial remodeling is any change in the size, shape, or function of the LV such that cardiovascular survival is altered. Although symptoms and LV ejection fraction are commonly used as surrogates because they are readily quantifiable, both are relatively poor indicators of survival [9]. Presently, only two classes of medications, namely, $\beta$-blockers and angiotensin-converting enzyme inhibitors, have been shown to significantly improve remodeling and survival beyond their effects on symptoms or blood pressure. By studying the remodeling process explicitly, the goal of our laboratory has been to identify additional potential mediators of regression, or beneficial remodeling.

We have shown the expression pattern of periostin in both experimental and clinical progression and regression of ventricular hypertrophy. Is periostin simply a marker of hypertrophy or does it have a formal role in the remodeling process? We recognize the observational limitations of our study and acknowledge that the changes in periostin may be a phenomenon occurring in parallel with changes in hypertrophy.

We believe, however, that periostin is more than an innocent bystander. Indeed, periostin is positioned as a key ECM protein that likely serves several beneficial roles in the development and maintenance of a well-functioning ventricle. We observed significant tissue levels of periostin in all animals. This is consistent with previous data demonstrating periostin is active developmentally in the endocardial cushion formation [5]. In addition, a recent study showed an increased rate of ventricular rupture after myocardial infarction in periostin knockout $\left(\mathrm{Pn}^{-/-}\right)$mice [10]. Collectively, these studies and ours demonstrate that periostin is an integral part of a well-functioning myocardium.

Teleologically, as with LVH, some periostin is good, whereas overexpression can allow for maladaptive LVH and heart failure. In pressure overload, periostin is highly over-expressed and is associated with numerous cardiovascular pathologies. Principally, there is an increased volume of collagen deposition and fibrosis within the myocardium and in perivascular tissues. Increased collagen and fibrotic proliferation is believed to impair ventricular compliance and lead to diastolic dysfunction [11]. This may have contributed to the decreased contractility that we observed in our pressure overload animals [7]. 
Correspondingly, the increased perivascular expression of periostin and collagen that we, and others, have observed is believed to impair nutrient and oxygen delivery to the myocardium [12]. It is likely that this further diminishes the ability of the myocardium to respond to the increased work of pressure overload.

Although we observed a diffuse increase in periostin expression and collagen deposition across the entire LV in our model, there was a significant predominance of expression in the interventricular septum. This may be due to regional differences in strain experienced by the $\mathrm{LV}$ component of the septum, a phenomenon well documented in humans but not yet in mice [13].

Importantly, in our series of regression experiments, we were able to successfully demonstrate that periostin expression decreased with relief of pressure overload. It is unknown whether the process of regression actively induced metabolism of periostin, or whether the natural tissue half-life of periostin is relatively short and dependent on continued expression. In contrast, collagen is believed to be a more durable product in fibrosis, and yet collagen was similarly less present in hearts that had experienced relief of pressure overload. This suggests that an active process within the ECM of the myocardium contributed to the metabolism of collagen. Previous authors have speculated that fibrosis and collagen deposition significantly lessen the ability of a ventricle to undergo regression of hypertrophy [14]. We believe our findings demonstrate that collagen-based changes in the ECM of the LV wall associated with LVH are, in fact, reversible.

Our echocardiographic studies demonstrated that 4 weeks of pressure overload resulted in increased chamber dimension and decreased LVEF, both of which contribute to heart failure [7]. In regression, we observed a reversal of both phenomena, indicating rescue. Although periostin is believed to be important in establishing and maintaining a healthy and functional ECM, it is likely that in the pathophysiology of pressure overload, it contributes to the dysfunction that leads to features of heart failure. Initial studies strongly linked gene expression of periostin with the progression of heart failure [4]. More recent studies with $\mathrm{Pn}^{-/-}$mice have demonstrated that if mice survive myocardial infarction, then the absence of periostin is associated with improved long-term myocardial function [10], suggesting that the presence of periostin may significantly impair recovery from an ischemic event.

In addition, studies of periostin in cancer have shown that periostin is highly associated with tissue invasion and metastasis. It is typically expressed at border zones, and periostin expression by tumor cells has been directly correlated with loss of host tissue integrity and tumor in-growth $[6,15]$. Although the mechanism of action remains to be determined, it is possible that the characteristics that lead to tissue invasion are similar to those that facilitate ventricular susceptibility to wall stress and pressure overload. Importantly, our findings, including those in our heart failure patients with bridge to transplant VADs, indicate that the LV dilatation and wall thickening associated with pressure overload are partly reversible and that this reversal occurs in an environment of decreased periostin expression.

We fully acknowledge several limitations of the study. Our mouse model of pressure overload and relief is most analogous to aortic valve replacement therapy for aortic stenosis. Our human model of pressure overload is necessarily quite different, in that there are no conditions in which heart tissue may be readily removed from healthy individuals. We believe that our current model, using pressure overloaded, failing hearts and then resampling after ventricular assist, is the closest human model available that affords ready access to tissue.

A related limitation is that the fibrosis that occurs in adult hearts from pressure overload is typically the result of longstanding pathology. In the mice, there is an acute onset of pressure 
overload, with a relatively rapid progression of hypertrophy and even failure. Despite these differences, both groups showed significant decreases in periostin expression with relief of pressure overload, further reinforcing our hypothesis that excessive periostin expression is associated with ventricular dysfunction and that functional improvement is associated with decreased periostin expression.

In summary, periostin is significantly over-expressed in conditions of myocardial pressure overload and significantly under-expressed in relief of pressure overload. These expression changes correspond closely with ventricular function, myocardial hypertrophy, and ventricular fibrosis. Although mechanisms of action are yet to be determined, a variety of reports suggest that periostin, although necessary developmentally, has significant adverse effects during pathologic over-expression. Specifically, ventricular remodeling is associated with greater dysfunction during periostin expression, and pressure offloading and functional improvement are linked with decreased tissue levels of periostin. We believe our data support further studies that target periostin as a means of therapy for fibrotic myocardial disease.

\section{Acknowledgments}

This work was supported by grants from the American College of Surgeons and by the Foundation for Thoracic Surgery Research and Education. We wish to acknowledge the technical support of Mauricio Rojas, MD, and editorial support of Margaret Alford Cloud.

\section{References}

1. Vakili BA, Okin PM, Devereux RB. Prognostic implications of left ventricular hypertrophy. Am Heart J. 2001; 141:334-41. [PubMed: 11231428]

2. Stansfield WE, Charles PC, Tang RH, et al. Regression of pressure-induced left ventricular hypertrophy is characterized by a distinct gene expression profile. J Thorac Cardiovasc Surg. 2009; 137:232-8. 8e1-8. [PubMed: 19154930]

3. Wagner RA, Tabibiazar R, Powers J, Bernstein D, Quertermous T. Genome-wide expression profiling of a cardiac pressure overload model identifies major metabolic and signaling pathway responses. J Mol Cell Cardiol. 2004; 37:1159-70. [PubMed: 15572046]

4. Blaxall BC, Spang R, Rockman HA, Koch WJ. Differential myocardial gene expression in the development and rescue of murine heart failure. Physiol Genomics. 2003; 15:105-14. [PubMed: 12865503]

5. Kruzynska-Frejtag A, Machnicki M, Rogers R, Markwald RR, Conway SJ. Periostin (an osteoblastspecific factor) is expressed within the embryonic mouse heart during valve formation. Mech Dev. 2001; 103:183-8. [PubMed: 11335131]

6. Kudo Y, Ogawa I, Kitajima S, et al. Periostin promotes invasion and anchorage-independent growth in the meta-static process of head and neck cancer. Cancer Res. 2006; 66:6928-35. [PubMed: 16849536]

7. Stansfield WE, Rojas M, Corn D, et al. Characterization of a model to independently study regression of ventricular hypertrophy. J Surg Res. 2007; 142:387-93. [PubMed: 17574596]

8. Abramoff MD, Magelhaes PJ, Ram SJ. Image processing with ImageJ. Biophotonics Int. 2004; $11: 36-42$.

9. Cohn JN, Ferrari R, Sharpe N. Cardiac remodeling- concepts and clinical implications: a consensus paper from an international forum on cardiac remodeling. Behalf of an international forum on cardiac remodeling. J Am Coll Cardiol. 2000; 35:569-82. [PubMed: 10716457]

10. Oka T, Xu J, Kaiser RA, et al. Genetic manipulation of periostin expression reveals a role in cardiac hypertrophy and ventricular remodeling. Circ Res. 2007; 101:313-21. [PubMed: 17569887] 
11. Zile MR, Brutsaert DL. New concepts in diastolic dysfunction and diastolic heart failure: Part I: diagnosis, prognosis, and measurements of diastolic function. Circulation. 2002; 105:1387-93. [PubMed: 11901053]

12. Baudino TA, Carver W, Giles W, Borg TK. Cardiac fibroblasts: friend or foe? Am J Physiol Heart Circ Physiol. 2006; 291:H1015-26. [PubMed: 16617141]

13. Boettler P, Claus P, Herbots L, et al. New aspects of the ventricular septum and its function: an echocardiographic study. Heart. 2005; 91:1343-8. [PubMed: 15761045]

14. Gao XM, Kiriazis H, Moore XL, et al. Regression of pressure overload-induced left ventricular hypertrophy in mice. Am J Physiol Heart Circ Physiol. 2005; 288:H2702-7. [PubMed: 15665058]

15. Shao R, Bao S, Bai X, et al. Acquired expression of periostin by human breast cancers promotes tumor angiogenesis through up-regulation of vascular endothelial growth factor receptor 2 expression. Mol Cell Biol. 2004; 24:3992-4003. [PubMed: 15082792] 

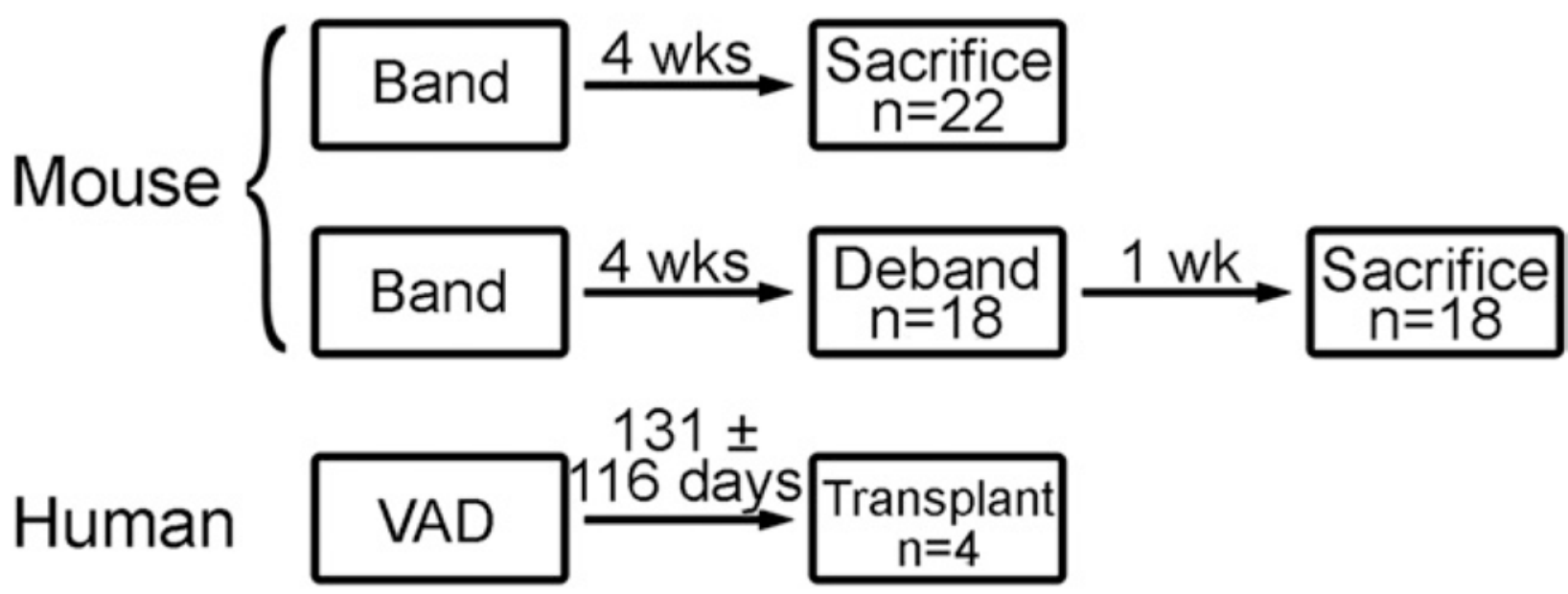

Fig. 1.

Overview of mouse experiments and timeline for human tissue procurement. (VAD = ventricular assist device.) 


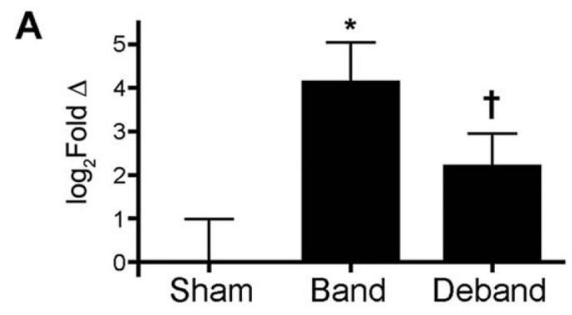

B

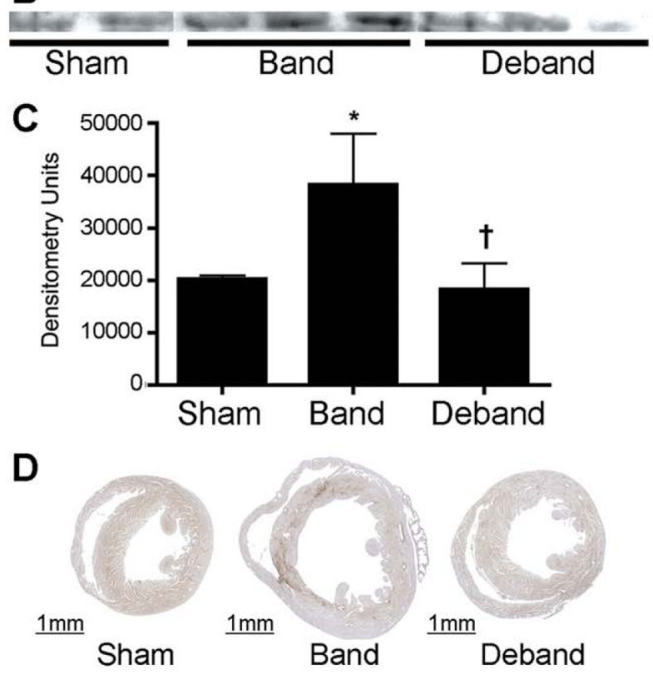

Fig. 2.

Periostin expression in experimental pressure overload and relief. (A) Mouse hearts were procured at the respective times with analysis for real-time polymerase chain reaction of periostin expression relative to $18 \mathrm{~S}$ rRNA $(\mathrm{n}=5,4$, and 4 for sham, band, and deband mice; *band vs sham, $\mathrm{p}=0.002$; ${ }^{\dagger}$ deband vs band, $\mathrm{p}=0.034$ ). (B) Western blot of periostin protein expression is corroborated by $(\mathrm{C})$ densitometry $(\mathrm{n}=2,3$, and 3 for sham, band, and deband mice; *band vs sham, $\mathrm{p}=0.042$; ${ }^{\dagger}$ deband vs band, $\mathrm{p}=0.038$.) The error bars in (A) and (C) reflect the standard error. (D) Immunohistochemistry is shown for periostin staining. 

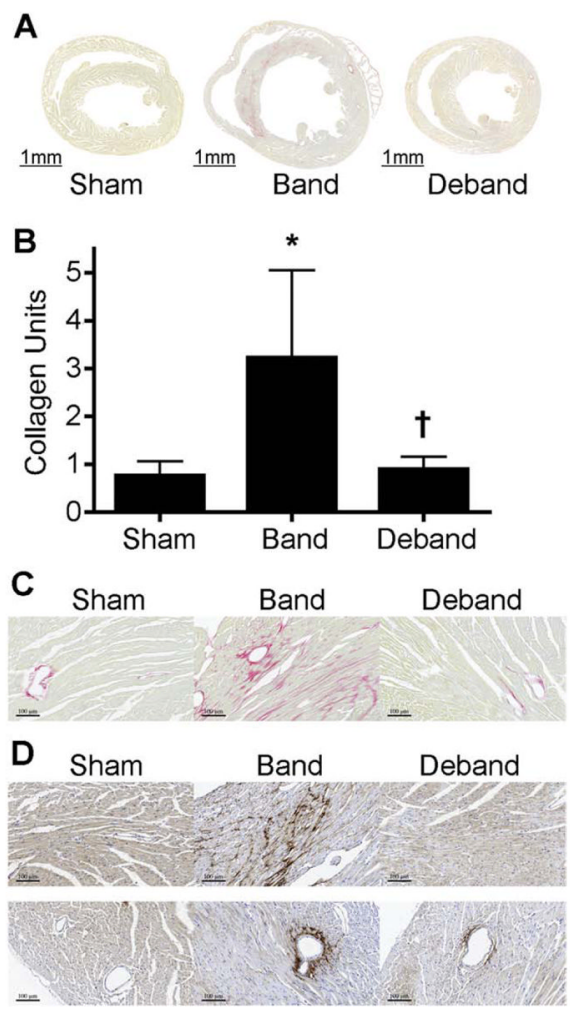

Fig. 3.

Periostin and fibrosis. (A) Mouse ventricles were stained with picrosirius red to detect collagen deposition at each respective time. (B) Immunohistochemical quantification of whole ventricle collagen staining $(\mathrm{n}=4,13$, and 9 for sham, band, and deband; *band vs sham, $\mathrm{p}=0.0001$; $^{\dagger}$ band vs deband, $\left.\mathrm{p}=0.0002\right)$. (C) Higher power $(\times 20)$ objective of collagen-stained sections. (D) Immunohistochemical staining for periostin expression. (Scale bar for $\mathrm{C}$ and $\mathrm{D}=100$ microns.) 

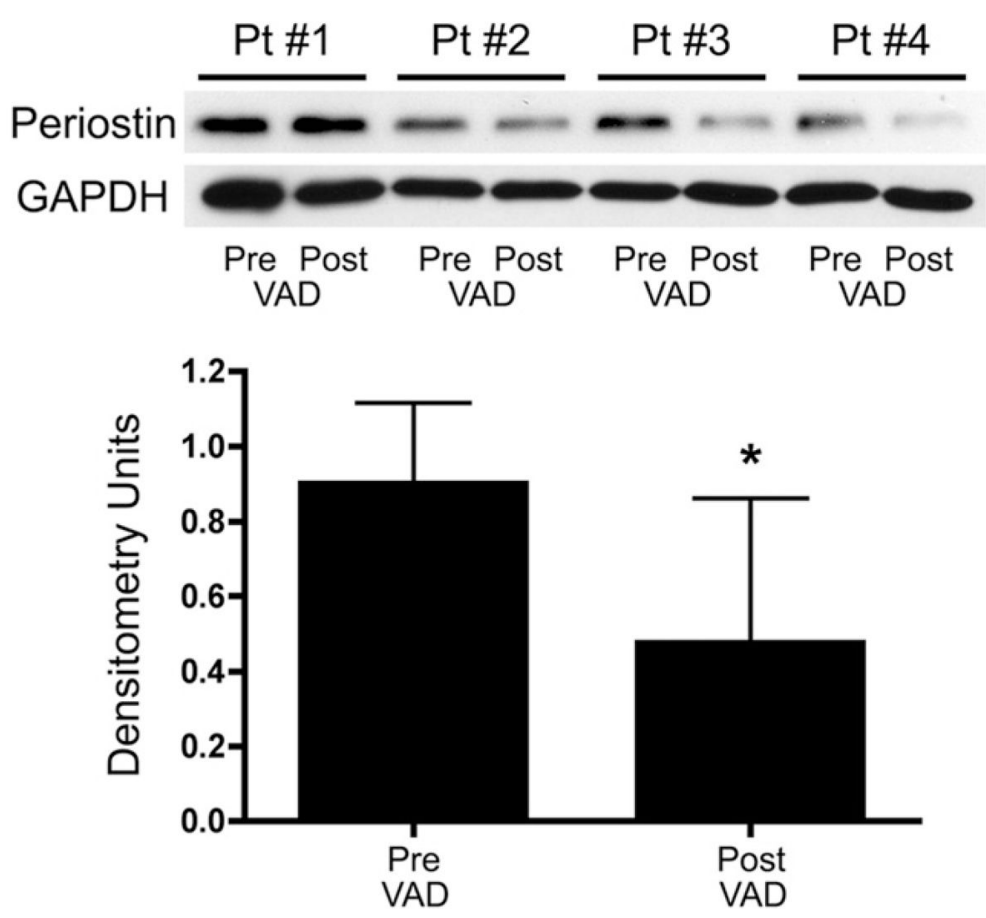

Fig. 4.

Western blot of periostin in matched human tissue samples before and after treatment with ventricular assist device (VAD). Densitometry of protein expression levels relative to glyceraldehyde-3-phosphate dehydrogenase (GAPDH; $\mathrm{n}=4$ paired samples; *post-VAD vs pre-VAD, $\mathrm{p}=0.077$ ). 


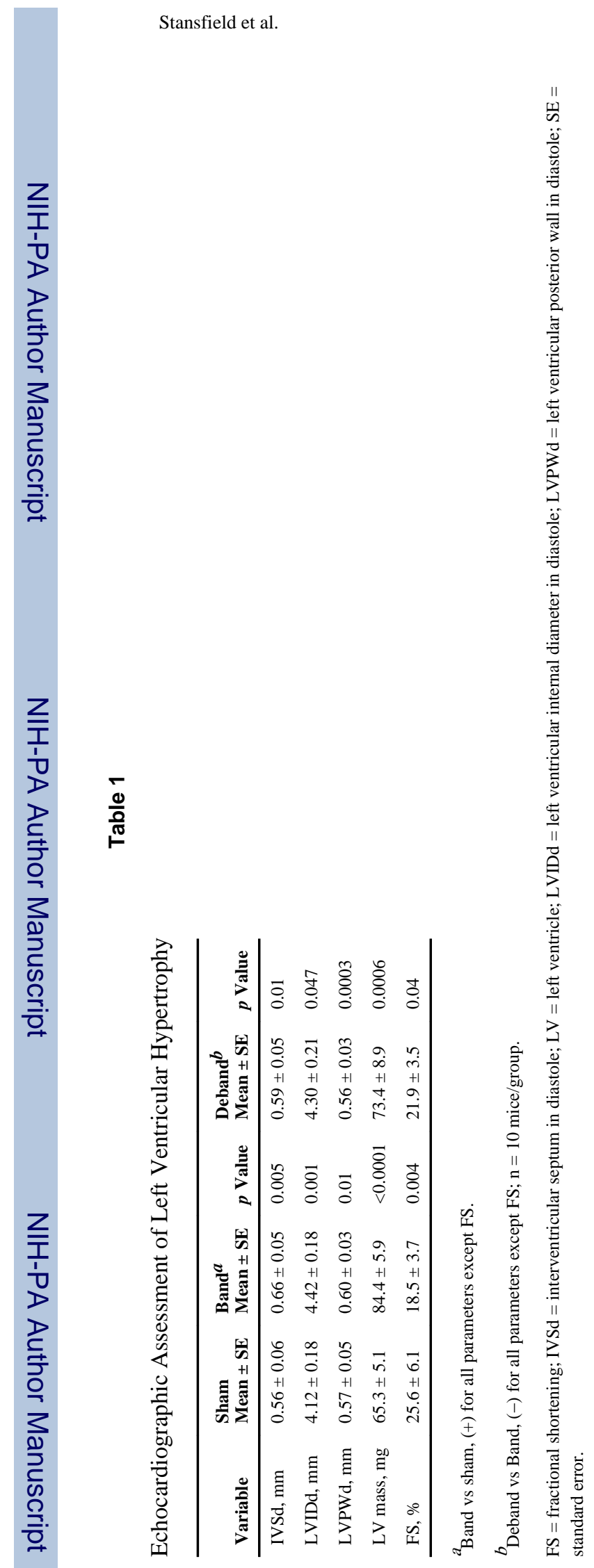

Ann Thorac Surg. Author manuscript; available in PMC 2013 June 19. 